\title{
IDEALI ETICI E PENSIERO POLITICO NEL DE RECUPERATIONE TERRE SANCTE (1306) DI PIERRE DUBOIS
}

Alessandro Ghisalbertit

SÍNTESE - Pierre Dubois, um dos legistas de Filipe, o Belo, em seu tratado Sobre a recuperação da Terra Santa produziu um texto que não possui grande importância filosófica. Entretanto, suas propostas politicas apresentam-no como um homem com algumas propostas interessantes, baseadas em Siger, R. Bacon e R. Lullio. A necessidade da paz, a reforma da Igreja, a unidade da fé, uma Europa de nações são alguns dos temas desenvolvidos.

\begin{abstract}
Pierre Dubois, one of the experts in law in the court of Philip IV (Philip, the Fair), in his treatise about the restoration of the Holy Land, has written a text without a great philosophical relevance. Nevertheless, in his philosophical proposals, he gives some interesting ideas based on Siger, R. Bacon and R. Lull. Thus, for example, he underlies the necessity of peace, the reformation of the Church, the unity of faith and the division of Europe into nations.
\end{abstract}

Singolare scrittore politico degli inizi del Trecento, Pierre Dubois è nato a Coutances, in Normandia, tra il 1250 e il 1255; discepolo a Parigi di Tommaso d'Aquino e di Sigieri di Brabante, "avvocato del re" sotto Filippo IV il Bello, è morto intorno al 1320.

Nei suoi scritti Dubois mostra una solida conoscenza del codice di diritto romano, per cui si suppone che egli abbia compiuto anche studi giuridici, che gli consentirono di svolgere la professione di avvocato. Ci troviamo di fronte a uno scrittore indipendente dal mondo accademico del tempo, nella condizione di difendere le proprie tesi in modo autonomo rispetto alle scuole ufficiali, non però senza limiti, che gli derivarono dall'essere expertus advocatus regalis, in un contesto di vicende politiche che avrebbero portato all'aspro conflitto tra papa Bonifacio VIII e il re di Francia Filippo il Bello.

La complessa personalità di Dubois è messa in luce dalle tesi centrali dei suoi due trattati più organici (le altre sette opere pervenuteci rivelano un carattere occasionale); nel primo di essi, intitolato Summaria brevis et compendiosa doctrina felicis expedicionis et abreviationis guerrarum ac litium regni Francorum, scritto negli ultimi mesi del 1300, egli propone una nuova tattica di guerra e progetta uno sveltimento della procedura giudiziaria, tale da porre rimedio all'ingerenza dei tribunali

- Università Cattolica del Sacro Cuore - Milano - Italia.

\begin{tabular}{|l|l|l|l|l|l|}
\hline VERITAS & Porto Alegre & v. 40 & $\mathrm{n}^{2} 159$ & Setembro 1995 & p. 643-658 \\
\hline
\end{tabular}


ecclesiastici nel campo della giurisdizione civile. ${ }^{1}$ Il secondo trattato organico, decisamente l'opera più significativa del Dubois, è il De recuperatione Terre Sancte, composto tra il 1305 e il 1307 e dedicato a Edoardo I d'Inghilterra, nella prospettiva di una più generale diffusione in Europa, ma in realtà destinato a Filippo il Bello, lettore privilegiato, per il quale è certamente stata scritta la seconda parte. ${ }^{2}$

Il movente politico dell'opera è costituito dalla prospettazione di una crociata destinata a un sicuro successo dei cristiani nella liberazione dei luoghi santi dagli infedeli, che, dopo la riconquista di S. Giovanni d'Acri nel 1291, erano tornati padroni di tutta la Terra Santa. La preparazione e lo svolgimento dell'impresa sono progettati in modo del tutto nuovo ed originale, e si sviluppano in una serie di riforme che tendono a riorganizzare l'assetto politico dell'Europa, la ratio studionum, la politica degli stati e l'organizzazione della chiesa. Prima e indispensabile condizione è la pace tra i cristiani, universale e sicura.

La singolare opera del Dubois testimonia l'estensione del dibattito dottrinale al di fuori dell'ambito accademico; la coscienza della cristianità reclamava pace tra i cristiani, per il raggiungimento della quale le nuove consapevolezze intellettuali, unitamente alle insorgenti conflittualità, postulavano precise e chiare regole. L'aspirazione a una pace duratura, leggibile anche come un apporto della nuova evangelizzazione inaugurata da Francesco di Assisi, può essere interpretata come segno di nuove aperture d'orizzonte sul piano della teoria politica, che i commentari alla Politica di Tommaso d'Aquino e di Sigieri di Brabante avevano contribuito a diffondere in occidente. L'incidenza della scienza politica aristotelica nei trattati di Dubois, unitamente alle numerose citazioni di altre opere dello Stagirita e all'esaltazione della logica, sono gli elementi basilari della sua ricerca di nuove frontiere e di nuove autonomie degli ambiti disciplinari.

\section{1 - La pace come valore etico e politico}

Il pensiero e il desiderio di una pace duratura, frutto di giustizia fra gli uomini, sono vivi e ricorrenti in tutti gli scritti del Dubois, ma nel De recuperatione Terre Sancte essi si propongono come l'urgenza storica in forza della quale l'autore può garantire una ferma motivazione alla plausibilità delle sue idee di riforma nel campo politico e in quello ecclesiastico.

La dedica del De recuperatione ad Edoardo I celebra la magnanimità del sovrano Plantageneta consistente nel mostrarsi disposto all'impresa crociata, per la buona riuscita della quale Dubois offre vari suggerimenti, ritenendone più che opportuna l'attuazione ai fini non soltanto del recupero della Terra Santa, ritornata sotto il dominio degli infedeli, ma anche della sua definitiva conservazione nelle mani dei principi cristiani.

1. Nel contempo l'autore trova modo di anticipare molte idee caratteristiche dei suoi trattati successivi, quali l'avversione al celibato ecclesiastico e al potere temporale di papi (il trattato è pubblicato dal Kämpf in Quellen zur Geistesgeschichte des Mittelalters und der Renaissance IV, Lipsia-Berlino, 1936.

2 Edito dal Langlois, Parigi 1891. 
La riconquista della Palestina è però subordinata al ristabilimento di una pace salda e strutturalmente indefettibile tra i principi cristiani, garantita alla radice da un rigoroso ordinamento, capace di risolvere ogni forma di attrito e di controversia giurisdizionale. Citando dal Vangelo, Dubois attribuisce valore politico fondamentale all'affermazione che "omne regnum in se divisum, desolabitur". L'impresa crociata esige uno sforzo economico e militare di ordine troppo grande per essere sostenuto in un clima di discordia tra i principi concorrenti; qualora infatti i singoli comandanti impegnati contro i Saraceni sapessero di conflitti scoppiati in loro assenza nei potentati di provenienza, non potrebbero non fare immediato ritorno nelle loro terre, lasciando sfumare nel fallimento l'impresa d'oltremare, "prout olim multociens contigit ibidem".

Dubois asserisce la necessità che tutti i cattolici, "saltem ecclesie romane obedientes", confermino la pace, in modo tale da favorire l'unità indivisibile della respublica christiana; contro le forze del male, che "habitant libentius in illa terra quam alibi", l'unità e la pace, precondizioni necessarie di una spedizione nei luoghi santi, possono essere concordate per diretto intervento del papa, "cum assensu generalis concilii omnium catholicorum principum et prelatorum". ${ }^{3}$

La deplorevole e rissosa belligeranza dei cristiani ostacola irreparabilmente le operazioni dei crociati: tedeschi e spagnoli, ad esempio, benché bellatores incliti, si sono già ritirati dall'impresa; le guerre tra cattolici, poi, sono cariche di aggravanti e portano molti alla dannazione. Con Aristotele Dubois afferma: "Omne bellum in se malum et illicitum; in tantum quod qui appetit bellum propter bellum in fine malicie est", ${ }^{4}$ e commenta che la sola guerra condotta per portare la pace può essere ritenuta lecita, come sostengono i giuristi.

La guerra dinastica crea le condizioni della vendetta, non della pace; e ciò ostacola lo svolgimento dell'unica guerra che potrebbe riportare la cristianità all'unità, cioè la crociata per il recupero della Terra Santa. "Omnis virtus unita fortior est seipsa dispersa et divisa", dice Aristotele, e i demoni malvagi conoscono questa verità: possedendo un potere divinatorio di molto superiore a quello di qualsiasi saggio anziano, in quanto hanno perfetta memoria di tutto il passato, operano attraverso i sentimenti di rancore per dividere quelle forze cristiane che potrebbero, una volta concordi, inferire loro un duro colpo riconquistando l'eredità del Salvatore.

Ammesso poi che le potenze cristiane riescano a coalizzarsi in unità, Dubois ritiene che contro le forze del male sia imprescindibile l'apporto efficace della preghiera della Chiesa, la quale a sua volta si rivela capace di sostenere un tale compito se si sottopone al suo interno ad una severa riforma.

L'unione tra le forze temporali e le energie spirituali può positivamente sprigionare l'ardorem salutis Terre Sancte, capace di sospendere ogni conflitto, demandando la soluzione delle eventuali controversie ad arbitri al di sopra delle parti, appositamente istituiti per sanzionare i contravventori dell'ordine giurisdizionale: "Nullus catholicus contra catholicos currat ad arma, nullus sanguinem baptizatum

3 In un altro passo, citando Phil. 4, 7. Dubois afferma che la richiesta della pace indirizzata a Dio ha per fine la perfezione della virtù e della conoscenza che non possono altrimenti essere acquisite.

4 De recuperatione, 2, p. 4 (Aristotele, Etica Nicomachea, X, 7 [1177 b 8-10]). 
effundat: quicunque preliare volentes, contra fidei christiane inimicos, Terre Sancte sanctorumque locorum Domini, non contra fratres, occasionem corporalis et spiritualis perditionis querendo, studeat preliare".

La pace si configura come la premessa necessaria alla riuscita dell'intervento crociato in Terra Santa, ma Dubois osserva che in quanto fine ultimo ed universale, ${ }^{5}$ essa, pur essendo l'intenzione primaria, data la sua anteriorità intenzionale cui secondo Aristotele corrisponde la posteriorità "in operis executione" ${ }^{\prime \prime}{ }^{6}$ implica prima di tutto la rimozione di tutti gli ostacoli che impediscono l'avvento e la risoluzione preliminare di qualsiasi elemento che verosimilmente ne occasioni l'impossibilità.

Per questo, come già accennato, Dubois entra nel merito di una capillare riforma del settore degli studi e di un'incisiva trasformazione dell'istituzione ecclesiastica.

\section{2 - La crociata come via per l'unificazione dei regni dell'occidente cristiano}

Nell'elaborare il suo progetto di pace, Dubois riporta in dettaglio alcuni suggerimenti di strategia e di logistica che a suo parere dovrebbero caratterizzare l'azione comune dei sovrani cattolici, nell'eventualità auspicabile di una generale coalizzazione contro gli invasori infedeli. Una crociata, infatti, costituirebbe una richiesta forte di coesione degli intenti politici dei principi .

Innanzitutto Dubois propone forme di punizione esemplare per tutti coloro che in qualsiasi modo contribuiscono a fomentare le guerre tra cristiani: sequestro dei beni, bando dalle loro proprie terre ed esilio coatto in Terra Santa, finanziato nella giusta misura con i loro averi confiscati, e finalizzato al ripopolamento e alla difesa dei territori appena conquistati. In questo modo, sostiene Dubois, le sanzioni si dimostrerebbero utili ed efficaci, perché toccherebbero direttamente lo stato sociale ed economico di coloro cui vengono comminate, pur figurando, di fatto, meno gravi di una pena spirituale quale la scomunica, che pregiudicherebbe eternamente e odiosamente la salvezza delle anime: "Pena temporalis, licet sine comparatione minor eterna, plus timebitur, plus proficit Terre Sancte; minus nocebit omnibus proximis et conjiunctis dampnatorum". ${ }^{7}$

In secondo luogo Dubois argomenta che, data la mole enorme di ciò che sarebbe necessario raccogliere per sostenere il trasporto delle milizie in Terra Santa, cosa pressocché impossibile per i singoli principi, sarebbe opportuno pensare ad un migliore sfruttamento dei beni posseduti e male amministrati dagli Ospitalieri e dai Templari.

5 Nell'ultimo paragrafo della prima parte dell'opuscolo, Dubois argomenta che la pace non è individuata quale fine universale soltanto a partire dagli studi teologici, ma anche, semplicemente, basandosi sul contributo dei massimi filosofi dell'antichità: "philosophi [...] irrefragabiliter concluserunt unum fore primum principium movens omnia [...] solum per se et de se ens, et bonum; per bonitatis cujus participationem et non alias, omnia causata recipiunt et participant bonitatem [...]; quod principium et causam primam Deum appellamus" (109, p. 94).

De recuperatione, 28, p. 21 (Anistotele, Etica Nicomachea, X, 7 [1177 b 8-10]).

7 De recuperatione, 4, p. 8. 
La Terra Santa, poi, avrebbe bisogno di un impiego di forze superiori a quelle inviate sul campo fino a quel momento, ragione per cui il papa, "hujusmodi negotium plurimum cordi habens", dovrebbe sollecitare i prelati ad inviare colà un congruo numero di combattenti, armati ed equipaggiati come conviene, e parimenti dovrebbe sollecitare i principi, che a loro volta, se impossibilitati ad impegnarsi in prima persona, dovrebbero delegare funzionari idonei per un'eventuale reclutamento di massa all'interno dei loro domini.

Si potrebbero così costituire alcune acies compatte per estrazione geografica e per comunanza di lingua, e per fare ciò l'idea del Dubois sarebbe quella di indurre uomini e donne di qualsiasi condizione ad inviare armati mediante una specie di movimento propagandistico, emotivamente coinvolgente, attuato con sfilate attraverso città, castelli e villaggi: "Sic per concursum et conflictum augebitur, ultra quam modo credi posset, numerus pugnatorum..$^{8}$ Principi e Grandi dovrebbero poi vincolarsi con la promessa di lasciare sul campo tutte le loro milizie con le convenienti provvigioni anche nel caso della loro morte, o di sopravveniente invalidità, 0 di qualsiasi altro motivo che li potesse indurre al rientro. Uomini e donne validi sarebbero indotti a procurare ogni anno quanti più armati possibile, anche, eventualmente, con le loro mogli, in modo tale da potersi addirittura stabilire oltremare per popolare la terra conquistata; qualche valoroso ed esperto comandante, infine, fermandosi a presidiare le nuove terre, potrebbe rappresentare ufficialmente il principe cattolico per cui presta servizio, e ogni regno dovrebbe preoccuparsi di costruire nelle terre occupate città, castelli e luoghi di accoglienza, in cui potrebbero via via trovare albergo i nuovi arrivati e gli invalidi, per ritrovare le forze necessarie a sostenere il combattimento.

A queste riflessioni seguono alcuni dettagli sulla ripartizione dei territori, sull'inquadramento delle milizie, sul modo di combattere ispirato alle tecniche dei Tartari e al metodo della "terra bruciata", e sulle diverse possibilità di avvicinamento verso la Terra Santa, per mare e per terra, da parte delle diverse compagini di truppe. 9

\section{3 - II rinnovamento degli studi}

Tra i requisiti indispensabili al recupero della Terra Santa, Dubois indica anche tutto ciò che genericamente concorre al benessere dei suoi nuovi futuri abitanti. A suo parere ciò troverebbe un sintetico riscontro nell'istituzione di scuole specificamente impostate per lo studio delle lingue. Ad un primo livello diverrebbe cosi possibile, per i coloni crociati provenienti da tutte le nazioni, trovare confessori esperti in ciascuna delle loro lingue; in seconda istanza, sarebbe opportuno ed utilissimo per i sovrani di Gerusalemme godere dell'aiuto di segretari capaci di comunicare nell'idioma degli arabi. Tra l'altro tale eventualità offrirebbe l'adito per una riunione alla Chiesa romana di alcuni popoli che, pur professandosi cattolici, tuttavia

8 De recuperatione, 16, p. 16. Più avanti (104, pp. 88-89) Dubois presenta l'idea di quattro grandi armate che per diverse vie, di mare e di terra, avrebbero dovuto avvicinarsi progressivamente alla Terra Santa, facendo del Mediterraneo un mare cristiano.

9 Questi argomenti, in parte, erano già stati trattati nella Summaria brevis. 
non obbediscono alla cattedra di Pietro e che, paradossalmente sembrano essere più numerosi degli stessi romani: "quod non posset esse nisi ecclesia romana plures bene literatos in eorum ydiomate haberet, per quos eis scriberet", ${ }^{10}$ visto che il primato romano esprime una precisa volontà di Dio, resa manifesta dagli statuti dei santi Padri, per i quali il pontefice romano è il capo della chiesa universale.

Allo scopo di ottenere l'unità vera della religione cristiana, Clemente V dovrebbe istituire in ogni provincia e presso ogni priorato dei Templari e degli Ospitalieri due o più scuole per fanciulli e fanciulle. Essi sarebbero dapprima istruiti nell'uso del latino, poi alcuni nella lingua greca, altri in arabo, altri nei diversi idiomi, soprattutto quelli dei popoli cattolici, affinché la Chiesa e i principi cattolici siano in condizione di comunicare con tutti gli uomini, attraendoli nell'unità della fede e del suo capo visibile.

Alcuni di essi verrebbero istruiti nella grammatica, nella logica, negli articoli della fede, nella sacramentaria, nel Nuovo e nell'Antico Testamento, e verrebbero inviati in Terra Santa per la cura delle anime. Altri, invece, verrebbero istruiti nella medicina e nell'arte veterinaria, in modo tale da garantire l'assistenza alla popolazione ed alle mandrie dell'esercito. Le fanciulle, poi, verrebbero istruite nella medicina, nella chirurgia e in quelle arti che ne costituiscono i preliminari, in modo tale che, cosi formate, potrebbero essere date in moglie - le più nobili e intelligenti, oltre che dall'aspetto attraente - ai principi, agli esponenti del clero orientale e ai maggiorenti più in vista, tanto nobilitate dallo studio e dalle doti da sembrare figlie di principi. Ciò, secondo Dubois, potrebbe addirittura servire a ravvicinare le chiese orientali a Roma, proprio attraverso l'abilità femminile nell'arte del persuadere.

Alla sommità del curricolo scolastico poi, per gli studenti più meritevoli e meglio disposti, si dovrebbero prevedere corsi di diritto, di astronomia, di scienze matematiche e naturali, di teologia e di medicina, al fine di preparare specialisti adatti per missioni ad alto livello diplomatico, "ut non esset qui posset sapientie romane ecclesie resistere". Tra l'altro, nota Dubois, una simile prospettiva offrirebbe all'occidente la possibilità di commerciare agilmente quei generi preziosi che si trovano in abbondanza nelle terre d'Oriente in cambio di ciò che invece comune e diffuso in Occidente.

La conoscenza delle lingue, infine, permetterebbe l'effettivo costituirsi di un'unica e solida monarchia spirituale, in grado di stabilire la sua potestà e la sua giurisdizione su tutte le terre cristiane, secondo la volontà del Signore che assicurò proprio il dono delle lingue ai primi evangelizzatori del mondo: "ad hunc finem quod procurarent omnes baptizari et credere, et Petro principi Apostolorum obedire, omnium credentium unam faciendam rempublicam". ${ }^{11}$

Per di più la fede cristiana potrebbe a poco a poco diffondersi anche tra i saraceni, attraverso l'opera discreta delle donne istruite e oculatamente date in spose a quei signori che, tra gli infedeli, vengono osteggiati dai correligionari; soprattutto, dice Dubois, sarebbero le loro mogli a desiderare la fede cristiana, mosse dal desiderio di entrare a far parte di un regime monogamico come le donne d'Occidente.

10 De recuperatione, 59, p. 48.

11 De recuperatione, 64, p. 55. 
Attraverso la pace e la concordia così ottenute tra tutti i cattolici seguirebbe per tutto il mondo cristiano un rinnovamento segnato dal progresso della virtù e della cultura, e dall'aumento della ricchezza e della longevità; i principi cristiani, non avendo più nulla da temere circa i loro beni e le loro terre, confluirebbero numerosissimi contro gli infedeli, determinati nell'idea di conservare e di amministrare in perpetuo la terra riconquistata: "Sic plus quam paulatim respublica catholicorum obedientium ecclesie romane fortiter augeretur, contra alios omnes rempublicam unam, et affectionem, amorem et caritatem ad Deum et proximum habentes". ${ }^{12}$

Alla diffusione e al consolidamento della monarchia cristiana contribuirebbe notevolmente lo studio della filosofia, nella quale dovrebbe istruirsi anche il fiore del personale militare, perché la storia tramandata dagli antichi insegna che facilmente a una conquista segue la fioritura culturale se opportunamente si provvede alla fondazione delle scuole nei luoghi più adatti. II regime degli studi elementari che, come già accennato, assorbirebbe i fanciulli in tenera età, dovrebbe prevedere un progresso delle discipline articolato a partire dalla lettura e dal canto del salterio, attraverso i grammatici e i classici, fino allo studio del Doctrinale del Villedieu e del Grecismum del de Béthume; successivamente, nelle fasi superiori, per i giovani si proporrebbe lo studio della logica sulle Summule in uso e delle lingue, poi l'approccio ai Naturalia attraverso un compendio dell'opera di Alberto Magno e alcuni estratti dall'opera di Tommaso d'Aquino, di Sigieri di Brabante e di altri dottori, l'avvio all'etica, alla retorica e alla politica, abbreviate in opere di compendio, alle leggi, al diritto civile e canonico, alla medicina, alla chirurgia e alle scienze matematiche.

Dopo l'abbozzo del programma di studio da attivare nelle scuole, Dubois si sofferma sulle procedure giuridiche, per le quali suggerisce un alleggerimento finalizzato ad accelerare la soluzione delle cause, per evitare le dannose conseguenze, in termini di guerre e di continue discordie, di troppo lunghe discussioni. Per il governo della Terra Santa riconquistata, allora, Dubois propone l'unificazione delle procedure giudiziarie, indipendentemente dalle regioni di provenienza dei contendenti; ciò a motivo del fatto che a quella terra, santificata dal sangue e dalle opere di Cristo, converrebbe più che ad ogni altra la pace, proprio perché più vicina e più amata dal re pacifico, e dunque sarebbe buona cosa che in essa più che in ogni altra si avvertisse la necessità di eliminare le discordie, una volta riconquistata ed abitata da cristiani. Considerato che qualsiasi discordia è male, e che tra due mali è doveroso scegliere il male minore, apparirebbe senza dubbio preferibile un exercitium litium planius et brevius; il sommo pontefice avrebbe allora il compito di stabilire in Terra Santa un ordinamento giuridico indipendente dalle tradizioni ormai gravose e lente.

12 De recuperatione, 70, p. 58. 


\section{4 - La riforma della Chiesa}

Parlando della possibilità di una spedizione per il recupero della Terra Santa, Dubois ha sottolineato la necessità del previo raggiungimento della pace in tutto l'Occidente europeo; ma la buona riuscita della Crociata, a suo parere, non dipenderebbe soltanto, come già si è accennato, dalla non belligeranza dei principi cristiani, dal momento che sarebbe assolutamente necessario il sostegno spirituale della Chiesa, unita e compatta tanto quanto, nella pace, la respublica christicolarum, cioè la compagine interstatale e sovranazionale della cristianità. L'unità della Chiesa nella preghiera si renderebbe indispensabile data la ferma opposizione delle forze del male che, secondo Dubois, infestano l'eredità di Cristo; tale unità non potrebbe però realizzarsi senza una profonda e rigida riforma delle istituzioni ecclesiastiche.

La Chiesa ha il compito di impetrare dal Dio degli eserciti la vittoria e la pace, perché le sole forze umane, "angelis malis contranitentibus", non sono in grado né di conquistare né di governare le terre di nuova conquista; occorre che si realizzino le condizioni profetizzate dal Salmo XXXII: "Declina a malo et fac bonum; inquire pacem et persequere ipsam", affinché, raggiunta la vera pace dei cuori, l'insieme universale di clero e popolo realizzi le condizioni descritte dagli Atti: "Multitudo credentium erat cor unum et anima una", ${ }^{13}$ e già descritte da Aristotele: "Omnis virtus unita fortior est seipsa diversa et divisa". ${ }^{14}$

Secondo Dubois, in vista dell'eliminazione dei conflitti, necessaria alla salvezza delle anime, è compito del sommo pontefice procedere ad una attenta analisi del comportamento di tutti gli ecclesiastici, perché prima di tutto tra di loro non scoppino guerre, liti e controversie a motivo, più che altro, della contesa dei beni temporali; gli ecclesiastici, infatti, sembrerebbero occuparsi molto di più delle faccende legate ai loro domini che non alla salute delle anime loro affidate, in contrasto con le stesse norme del diritto civile, che vuole che in ogni caso le anime siano preferite a qualsiasi altra cosa.

Spesso, nota Dubois, i prelati impegnano nella difesa di affari temporali molto più tempo e più denaro di quanto non ne mettano a disposizione della cura delle anime loro affidate; spesso i canonici nominati vescovi, subito dopo l'elezione, intentano cause per liti secolari e dedicano la loro attenzione ad esse molto più di quanto non facessero da semplici preti; spesso poi i vescovi scelgono un tenore di vita eccessivamente raffinato. La situazione morale della gerarchia ecclesiastica, commenta, è ben rappresentata dalle parole del Signore, quando dice: "Super cathedram Moysi sederunt scribe et Pharisei: que dicunt facite, sed que faciunt, facere nolite" ${ }^{15}$ ed anche dalle parole del Filosofo, che qualificano chi parla bene e agisce male come un falso maestro.

Una corruzione analoga a quella del clero secolare è diffusa anche negli ordini monastici. Gli abati benedettini, che non potrebbero possedere nulla senza macchiarsi di colpa grave, sono invece molto ricchi e cercano in ogni modo di accapar-

Atti, 4, 32 .

Mt. 7, 16. 
rarsi amministrazioni dentro e fuori le abbazie. Ciò comporta, ad esempio in Borgogna, che i figli dei ricchi principi abbraccino la vita religiosa per poter vivere comodamente nel lusso e nella lussuria.

Il papa dovrebbe considerare quante e quali guerre, e quanto pericolose, siano state intraprese anche dai suoi predecessori esclusivamente per la difesa del patrimonio di Pietro, e quanto grande sia il numero dei cattolici scomunicati soltanto per il loro tentativo di penetrare in quei territori. Inoltre dovrebbe tener presente il volume di atti simoniaci che si consumano nella normale amministrazione dei beni ecclesiastici, per non parlare di diversi cardinali, i quali sono costretti a vivere de rapina, dal momento che non vengono trattati in modo congruo economicamente.

"Peccata et occasiones delinquendi", conclude Dubois, "[...] ex nunc in posterum extirpandas, ne inde nascantur injurie unde jura nasci debent". ${ }^{16}$ In conseguenza di tali comportamenti all'interno del popolo cristiano sorgono gravi scandali. Per la riconquista e la conservazione della Terra Santa occorrono invece le orationes devote dei prelati, dei chierici e del popolo, perché le forze umane non possono bastare; ma se i vertici della Chiesa sono corrotti, probabilmente anche il popolo, proprio per l'esempio dei superiori, è portato alla corruzione.

Il papa Clemente, nella misura in cui avrà riformato lo stato dei prelati e dell'intera respublica christianorum, potrà elevare suppliche affinché le forze spirituali e temporali, unite come conviene, militando devotamente, possano ottenere una sicura vittoria contro gli infedeli.

In termini concreti una riforma globale della Chiesa può avvenire attraverso la rinunzia alla gestione diretta del patrimonio di S. Pietro, che ostacola inevitabilmente il sommo pontefice nel suo compito spirituale. Valutate le rendite, le entrate e le uscite, dedotti spese e oneri, tutti i beni del patrimonio dovrebbero essere affidati in perpetuam amphiteosim a qualche re o a qualche principe, che sarebbe comunque tenuto a garantire in perpetuo al papa un congruo reddito annuo. In tal modo il papa, attore e promotore della pace, non potrebbe essere sospinto a scendere in guerra a motivo della difesa del suo patrimonio, e potrebbe dedicarsi interamente alla preghiera, alle elemosine, alla contemplazione, alla lettura e alle dottrine scritturistiche, procurando la pace autentica per tutti i credenti in Cristo: "Sic sanctissimus papa thesauros non studebit aggregare, nec a spiritualium sollecitudine debita retrahetur; vitam ducet contemplativam, et activam favente misericorditer bonorum omnium largitore. ${ }^{17}$

Dubois prospetta una riforma anche del collegio cardinalizio, tale da garantire ai suoi membri una rendita tratta dallo stesso patrimonio di S. Pietro e adeguata alla loro condizione; ciò dovrebbe porre fine alle interminabili liti tra gli elettori e gli aspiranti alla curia per il mantenimento dei privilegi connessi alle cariche. Inoltre l'avvocato di Coutances prevede una cospicua confisca di beni ecclesiastici a favore della Terra Santa, per la sua completa riconquista e per la conveniente fortificazione; i beni temporali dei prelati dovrebbero essere amministrati da incaricati sicuri e idonei, fatta sempre salva la congrua rendita annua per lo svolgimento delle mansioni spirituali. 
Attraverso il complesso di tali provvedimenti, la respublica totius sancte religionis christicolarum si prefiggerebbe un unico fine, ricercando unicamente quello e disponendosi in modo tale da eliminare qualsiasi inclinazione dissonante nei suoi confronti; come un esercito, il mondo, così uniformato, tendendo ad un unico fine sotto un unico comandante, otterrebbe certa vittoria.

D'altra parte, argomenta Dubois, questi provvedimenti di riforma economica della Chiesa trovano ampio appoggio anche nella Scrittura, ove si dice che Giosuè stabili che la spartizione della terra promessa avvenisse tra undici delle dodici tribù di Israele, vincolate a mantenere la dodicesima, la stirpe sacerdotale di Levi, attraverso il pagamento da parte delle altre delle decime di tutti i raccolti; questo, affinché $\mathrm{i}$ leviti fossero disimpegnati per potersi dedicare interamente al culto.

Le misure applicate al clero secolare dovrebbero essere estese anche nei confronti dei prelati appartenenti agli ordini religiosi, e analoga regolamentazione, fatte le debite differenze, dovrebbe applicarsi anche agli ordini regolari femminili, per snellire le comunità sovraffollate, rendendo più proficua e più sana la convivenza monacale.

A riguardo del celibato ecclesiastico, infine, Dubois ritiene che sarebbe saggia l'abolizione, dichiarandosi convinto che la condizione di astinenza perpetua può risultare paradossalmente un incentivo alla tentazione, postulando una capacità di resistenza alle inclinazioni naturali non sempre conciliabile con la debolezza dell'uomo.

\section{5 - Il compito della Francia nella costruzione della pace}

Nella seconda parte dell'opuscolo, apertamente dedicata a Filippo IV il Bello, Dubois si impegna a trarre alcune conclusioni sugli argomenti variamente discussi nella parte precedente, per mettere in evidenza i ruoli e i vantaggi che in modo particolare potrebbero interessare la Francia a seguito del raggiungimento della pace e dell'applicazione delle diverse riforme proposte.

Nel suo progetto di pace, Dubois assegna alla Francia un ruolo centrale e predominante nella respublica christiana, motivato, a suo parere, da diverse ragioni, a cominciare dall'influsso favorevole dei corpi celesti e delle ottime condizioni climatiche, che fanno sì che tutti coloro che nascono sul suolo francese "melius sunt compositi, ordinati et complexionati quam aliarum regionum homines". ${ }^{18}$

La Francia era indiscutibilmente la maggior potenza demografico-militare del tempo, e poteva vantare per il suo sovrano una diretta discendenza da Carlo Magno, cosi da rivestire un'immagine di singolare autorevolezza nel contesto della cristianità. Essa poteva esser considerata come la nazione prediletta dal cielo, centro di espansione della fede e osservatorio privilegiato della salute della chiesa, a partire dal suo primo rappresentante, il vicario di Cristo. Dubois precisa che, una volta sedate le guerre, grazie alla tutela francese, il sommo pontefice potrebbe stabilirsi

18 De recuperatione, 139, p. 129. Nella Summaria cosi argomenta: "Expediret totum mundum subjectum esse regno Francorum, dum tamen rex ejusdem, prout fieri consuevit, in eodem regno generetur, nascatur, educetur et instruatur, et hoc propter astronum meliorem aspectum et influenciam quam dictum regnum habere percepimus [...] Gallici quidem longe certius utuntur vero judicio rationis quam alie quelibet nationes". 
definitivamente oltralpe, "cessantibus Romanorum et Lombardorum insidiis venenosis", per dedicarsi in tutta tranquillità al governo delle anime lontano dalle intemperie del clima italiano. ${ }^{19}$ Dopo gli innumerevoli abusi perpetrati dal papa, in quanto romano, contro la stessa potestà papale, i romani cederebbero finalmente la carica pontificia a un popolo che non ne avrebbe offeso l'onore e che permetterebbe al papa di vivere e di governare come vero Cesare, nell'esercizio pieno delle sue mansioni, che Dubois supporta con il riferimento al precetto di Cristo: "Reddite que sunt Cesaris Cesari"..$^{20}$ Inoltre il papa in Francia potrebbe liberamente creare cardinali francesi, trasferendo definitivamente la curia, e sottraendola, ancora una volta, alle mani rapaci dei romani.

Il raggiungimento della pace tra i cristiani, in secondo luogo, insieme alle riforme previste nella prima parte dello scritto, potrebbe arrecare enormi vantaggi alla Francia, anche attraverso un generale riassetto di molte regioni occidentali. Al re di Sicilia sarebbe eventualmente attribuito il regno di Gerusalemme, patrimonio ben più ricco dei suoi possedimenti precedenti; il re di Germania potrebbe definitivamente assicurarsi il dominio del suo regno e garantirsi il titolo imperiale, per sé e per i suoi discendenti, mentre Carlo di Valois sarebbe candidato per il titolo di imperatore dell'Impero Latino di Oriente. Tutto ciò, commenta Dubois, prospetterebbe uno sviluppo proficuo per il re di Francia e per i suoi discendenti, in quanto, una volta realizzatesi le previsioni sopraesposte, tutti i sovrani obbedienti alla chiesa di Roma si sottometterebbero in un certo senso alla sua autorità, confederandosi con Carlo di Valois, divenuto imperatore d'Oriente.

Sarebbe poi molto vantaggioso per il sovrano di Francia assicurarsi in eredità, attraverso un'azione diplomatica, alcuni territori della Germania situati al di qua del Reno e tra la Provenza e la Savoia, garantendo per contro al re della Germania la corona imperiale. Anche il papa, come si è visto, verrebbe a trasferire tutto il patrimonio della chiesa, compresi i potentati ad essa assoggettati nell'obbedienza vassallatica, nelle mani del sovrano francese, il quale potrebbe delegare per il diretto governo delle sostanze ecclesiastiche un figlio o un fratello, previamente creato senatore romano. Con ciò, sempre attraverso accordi diplomatici e nel rispetto della pace, si otterrebbe l'obbedienza dei re di Inghilterra, di Aragona e di Maiorca, attraverso la loro sottomissione al pontefice romano, e si stabilirebbe una convenzione analoga per cui all'usurpatore di Castiglia sarebbe in cambio affidato il regno di Granada, una volta ripresa ai Saraceni, obbligandolo all'obbedienza mediante il forte presidio militare francese, necessario per la resistenza agli eventuali attacchi degli infedeli. Infine, come già si è accennato, la coalizione delle forze dei sovrani occidentali dovrebbe riconquistare Costantinopoli per costituirvi l'Impero d'Oriente, risultato stategico di capitale importanza per costituire sulle coste del Mediterraneo un'ininterrotta catena di principati cristiani.

In re francese, naturalmente, stante la sua preminenza e il suo prestigio, non dovrebbe impegnarsi direttamente nelle campagne militari e non dovrebbe esporsi inutilmente al pericolo; ${ }^{21}$ delegando un congiunto per le rischiose operazioni milita-

19 Dubois non cela la sua disistima per l'Italia e gli Italiani, nifacendosi ai contrasti intervenuti poco tempo addietro tra la curia romana e la corte francese.

20 Mt. 22, 21. 
ri, egli deve piuttosto dedicarsi, nella tranquillità, alla procreazione, all'educazione e all'istruzione dei figli, all'esercizio della giustizia e alle provvigioni necessarie per la guerra, secondo il consiglio di Aristotele: "Homines intellectu vigentes naturaliter sunt aliorum rectores et domini". II principe non deve esporsi direttamente al pericolo della morte, perché non accada che, "percusso pastore, disperdantur oves gregis". 22

\section{6 - Il pensiero etico-politico di Dubois e le sue fonti}

Dubois si presenta come un autore non facile, che sembra resistere ad ogni definizione sintetica. Non è propriamente un filosofo, ma non gli si può rifiutare importanza nella storia delle idee o delle opinioni, sollecitato dall'intima esigenza di chiarimento dei principi, anche se non intende innalzarsi alla sfera delle teorie. Potrebbe dirsi più che altro un recettivo, con una sensibilità pronta a comprendere $\mathrm{e}$ quasi a indovinare, capace però soltanto di raccogliere senza dare seguito a una rielaborazione sostanziale.

Se difetta di attitudine speculativa, l'avvocato di Coutances è ricco di immaginazione, il che gli consente una totale risistemazione con la fantasia della realtà sociale e politica. Sul terreno del progettuale, dunque, va posta in generale e in fondo la sua visione politico-sociale, nonostante la sua pretesa di essere concretissimo e pratico nel dare suggerimenti di immediata attuazione. Perciò, riprendendo le valide analisi di M. Delle Piane ${ }^{23}$ si potrebbe parlare di mancanza di sistematicità, da una parte, ma anche, dall'altra, di una non indifferente apertura del suo pensiero verso la modernità.

I fondamenti del pensiero di Dubois sembra possano riconoscersi come pienamente medioevali: da una parte egli ha una profonda visione unitaria della società, dall'altra distingue nettamente due poteri derivanti da Dio, unificabili nella figura di Cristo re e sacerdote, entrambi necessari all'unum regnum. Il suo sforzo consistette nell'adattare questi principi ad una realtà storica in rapida evoluzione.

Dubois mantenne ferma l'idea dell'unica società cristiana: senza questo elemento, infatti, non sembrerebbe possibile intenderne il pensiero, pur ispirato, non senza oscillazioni, ad un nuovo concetto di ragione dello stato nazionale francese, contrapposto agli universalismi tradizionali. Si potrebbe dire che egli tentò faticosamente di conciliare l'affermarsi delle realtà particolari nell'ordinamento europeo con la convinzione di una persistente unità di base, fondata nella respublica christiana.

Dubois non avverti il bisogno di approfondimenti teorici e il suo interesse fu esclusivamente orientato alla politica pratica, pur non potendo prescindere dai principi e dalle convinzioni. Egli ricorse all'idea di una "società delle nazioni", di una federazione cristiana di stati sovrani, nella quale potremmo vedere adombrata ante litteram l'Europa delle nazioni, e ipotizzò, nutrito delle tendenze imperialistiche ed universalistiche della glossa al Diritto romano, un impero immaginario

21 Era ancora fresco il ricordo di Luigi IX e di Filippo l'Ardito, entrambi deceduti per cause fortuite proprio durante campagne militari.

22 Le stesse cautele dovrebbero valere per il figlio del re, se unico.

23 Cfr. M. Delle Piane, Vecchio e nuovo nelle idee politiche di Pietro Dubois, Le Monnier, Firenze 1959. 
come supremo potere internazionale, un superstato cui tutti i singoli Stati avrebbero dovuto essere sottoposti.

L'idea dell'unità, basata sulla reinterpretazione cristiana dell'universalismo antico, affiorava nell'antichità, prescindendo dalla elezione di Israele, anche attraverso la voce dei filosofi che concludevano irrefragabilmente all'unicità del fine e del motore, bene supremo, Dio reggitore, fondatore di pace e padre. Per Dubois, il munus della respublica christiana è quello di realizzare tale unità, e, nella sua prospettiva, il fine è Dio, concretamente e precisamente individuato nella pace. In tal modo, attraverso la ripetizione dell'argomentazione aristotelico-tomista, egli esprime un sentimento ed afferma un concetto comune al suo tempo: l'aspirazione profonda dell'uomo alla pace.

L'idea medievale della pace è un prodotto del cristianesimo, non solo come riporto teologico, ma anche come sentimento evangelico, e come esigenza scaturente dalla tormentata condizione politica. Il concetto medioevale di pace non si esaurisce nella sola idea del cessato uso delle armi o di un blando stato di quiete; esso si lega strettamente ai principi metafisici di unità e di finalità dell'universo (ordinatio), per cui la pace può corrispondere soltanto a realizzazione dell'ordine universale creato, di cui l'ordine civile è parte integrante.

La pace per Dubois non può non essere giustizia, d'altronde essa è ripetutamente dichiarata necessaria funzionalmente all'acquisto di virtù e di conoscenza, donde può sorgere in extremis l'unica giustificazione della guerra, lecita soltanto se istitutrice di pace. Nel desiderio di pace di Dubois tuttavia è anche possibile scorgere qualcosa di "moderno". La crociata infatti non era idea e operazione accessoria, ma si poneva come coronamento dello sforzo di pacificazione; essa poteva allargare l'idea e la realtà dell'ordo cristiano, fino a pensarlo coestensivo con il mondo in cui l'ecclesia universalis poteva ancora coincidere con la respublica christiana; parimenti, l'idea della pace non era in Dubois un argomento di maniera messo innanzi per coprire ben altra motivazione. Anche l'egemonia della Francia di fatto non era pensata che come necessaria all'ordine della cristianità, non solo per realismo politico e calcolo delle forze sul campo, ma per ragioni superiori di natura religiosa e, a suo modo, giuridica, squisitamente medievali. Solo il rex christianissimus ha diritto alla preminenza finalizzata all'ordine; e ciò aiuta a capire il perché allora lo si identificasse con il sovrano di Francia.

La modernità di Dubois non si misura sul parametro del nazionalismo, ma sul fatto che egli ritiene opportuno enumerare una serie di vantaggi temporali che conseguirebbero dal benefico raggiungimento della pace, offrendo uno stacco pragmatico dall'orizzonte di trascendenza inteso come unico riferimento per fondare la politica.

Per prima cosa la pace sarebbe importante per potere realizzare una globale riforma della società, presentata in funzione della riconquista e della gestione della Terra Santa, ma di fatto progettata anche indipendentemente da quel fine per un generale miglioramento della vita. In secondo luogo la civiltà cui Dubois aspira non si comprende facendo semplice riferimento alla cifra nazionalistica, ma esige forse l'idea ricorrente della elezione divina, trasferita, per così dire, da Israele alla Francia. Infine, dietro l'ideale della pace sembra trasparire, concretissima, un'istanza 
economica, che mira all'espansione del mercato. É un motivo poco più che accennato, ma detiene un enorme valore indicativo, per il fatto che l'idea economica diventa una componente essenziale dell'idea di pace.

Su un altro fronte, quello dei rapporti tra politica e istituzione ecclesiastica, nel Dubois sopravvive a pieno titolo l'idea dei due grandi istituti universalistici e viene mantenuta la prerogativa dell'auctoritas sacrata pontificum. Si può affermare che in Dubois è rispecchiato il permanere dell'universalismo spirituale, mentre va inesorabilmente attenuandosi l'idea imperiale di fronte alla presa di coscienza dell'immensità dell'Oriente. Si manifesta la consapevolezza che l'universalità cattolica è realizzabile soltanto nel quadro di uno sforzo volontario comune, impegno pratico convinto e adeguato, in concreto realizzato attraverso lo studio delle lingue orientali ed il conseguente confronto comparato tra culture.

Dubois ritiene che il passo più adatto da compiere per ottenere l'estensione del potere spirituale sia la convocazione di un concilio generale a Tolosa, senza però lasciar intendere che ciò comporti un'identificazione in esso della suprema autorità della Chiesa. Il primato del papa rimane indiscusso, e il papa resta princeps e più generalmente summus salutis reipublice curatus in terris. Su di lui ricade il dovere della reformatio status universalis ecclesie e della respublica christiana.

Per quanto poi concerne i rapporti tra papato e regno di Francia, come si è accennato, sembra riaffacciarsi l'idea veterotestamentaria dell'elezione divina applicata nell'identificazione della civiltà francese con la civiltà della repubblica cristiana. Ogni danno alla Francia, allora, è un danno anche per la Chiesa, e il pontefice ha dunque l'obbligo di cooperare all'affermazione della nazione prediletta, un impegno nell'ottemperanza al quale si misura la stessa bonitas papale. In sostanza Dubois sembra mirare ad un condominio sulla Chiesa nella ripartizione collaborativa tra papa e Filippo IV. L'appoggio alla politica francese, nell'intento del Dubois, sarebbe il compimento di tutta la riforma ecclesiastica da lui pensata, pur nel rispetto dei principi tradizionali. Il re di Francia è visto a sua volta come protettore speciale della cristianità, tenuto a custodirne l'ortodossia religiosa con un potere non derivato dal campo spirituale. Poiché la religione risulta essere un interesse di popolo, lo stato non ha soltanto il diritto, ma anche il dovere di intervenire in materia.

L'idea della riforma globale della Chiesa, basata sul principio della completa secolarizzazione dei beni ecclesiastici patrimoniali e del divieto del possesso dei beni produttivi, aveva radici evangeliche, ma sembra motivata anche dall'esigenza di ridurre l'autonomia e la potenza finanziaria e politica del clero a favore della Francia, cui spetterebbe il compito, a suo modo religioso, di governare in temporalibus la cristianità.

Si possono rintracciare in Dubois chiari riferimenti a pensatori eminenti del tredicesimo secolo che guadagnarono un posto speciale nella storia del pensiero medievale e ai quali il suo pensiero filosofico è debitore. Il punto decisivo di incontro tra questi autori è costituito dall'opposizione al pensiero tradizionale, da una analoga inclinazione alla critica e dall'accento posto sull'idea del progresso. I contemporanei che sembrano avere direttamente influito sul pensiero di P. Dubois sono Sigie- 
ri di Brabante e Ruggero Bacone, mentre Raimondo Lullo ha forse lasciato un'impronta più debole, quantunque non priva di significato.

1) Nel De recuperatione la figura di Sigieri, di cui espressamente Dubois si dichiara discipulus, viene elogiata ${ }^{24}$ e il suo legame con l'aristotelismo sigieriano traspare soprattutto nello sforzo di ricercare la verità attraverso lo studio e nel desiderio di comunicarla agli uomini. La forte stima da una parte della veritas e dall'altra della ratio diventa un percorso caratteristico della sua argomentazione; cosi egli sottolinea il proprio amore per la verità e il suo interesse a farla trionfare attraverso la ricerca e la persuasione in tutte le azioni, anche nella vita politica. ${ }^{25}$

Dubois però si accosta all'aristotelismo radicale di Sigieri soprattutto con la sua generale opposizione alla politica del papato, cioè alla dottrina della preminenza del potere della Chiesa su ogni altro potere. Egli auspica la distinzione del governo civile, cui sono affidati gli interessi temporali, il mantenimento della pace, dell'ordine e della giustizia, dalla giurisdizione ecclesiastica, esclusiva custode delle cose spirituali, liberata da qualsiasi forma di governo temporale. In questo modo disegna uno stato autonomo nell'organizzazione della vita terrena, discostandosi dall'idea prevalente nell'agostinismo politico medievale, che non attribuiva un fine proprio al potere civile, subordinandone l'esercizio alla finalità ultraterrena.

Dubois riprende da Sigieri anche l'idea della rilevanza delle leggi, giudicate l'unico strumento sicuro per il buon funzionamento del governo, al di là della probità degli amministratori. ${ }^{26}$

2) L'influsso di Ruggero Bacone ${ }^{27}$ fu molto importante per la formazione intellettuale di Dubois, soprattutto nell'affermazione dell'altissimo valore metodologico dell'esperienza, sfruttata al di fuori dell'impianto scientifico scolastico, per la sua utilità finalizzata a scopi specifici. Egli chiama l'esperienza suprema rerum magis$\operatorname{tra}^{28}$ e sostiene che bisogna apprendere dalle esperienze fatte; sulla base dell'esperienza consiglia al re di non impegnarsi nelle pericolose campagne militari, ammira la saggezza dei predicatori che viaggiano e si arricchiscono di nuove esperienze ed egli stesso si richiama, nella giustificazione delle sue proposte per la riforma della giustizia, alle proprie esperienze di avvocato.

Con Bacone Dubois è d'accordo anche sul tema del progresso e dello sviluppo delle scienze; egli censura l'arroganza di chi ritiene migliore e onnicomprensiva la disciplina in cui è specialista e sostiene che nessuno può presumere di essere nello stesso tempo dotto e versato in parecchi campi del sapere. Inoltre, come già Bacone, egli si dichiara convinto del fatto che, al di là della guerra, il modo più proficuo per penetrare in Oriente e per convertirlo all'obbedienza nei confronti della cristia-

24 De recuperatione, 132, p. 121: "Ad hec facit id quod super Polytica Aristotelis determinavit precellentissimus doctor philosophie cujus eram tunc discipulus, magister Segerus de Brabancia".

De recuperatione, 136, p. 126: "Tamen hec scribere volui, sequens doctrinam Philosophi qui ait quod: Omnes professores veritatis plus debent adherere veritati quam amicitie" (Aristotele, Etica Nicomachea, I, 6 [1096 a 15]).

26

27

28

Ibidem.

Dubois non ha mai indicato se abbia conosciuto personalmente Bacone all'università di Parigi.

De recuperatione, 76, p. $64 ; 135$, p. 124 : "Certum est, ut ait Philosophus, quod 'prudencia mondi hujus', quam asserit fore reginam omnium virtutum moralium, quoniam omnes in se complectitur, 'in nobis adquiritur per experienciam' (Aristotele, Etica Nicomachea, VI, 8 [1142 a 12]); "experiencia vero agibilium humanorum longo indigt tempore". 
nità romana sia lo studio delle lingue orientali, canale indispensabile di relazioni e piattaforma necessaria per una completa riorganizzazione dei corsi di studio.

3) Si può sicuramente affermare che Dubois conobbe a fondo il pensiero di Raimondo Lullo, in sintonia con il quale dimostra un forte ottimismo circa la buona riuscita delle ipotizzate riforme politico-ecclesiastiche. Dotati di grande capacità immaginativa, Lullo e Bacone hanno elaborato impegnativi progetti, cercando sempre di stabilirne minuziosamente la realizzabilità pratica; l'enorme desiderio missionario che contrassegnava Lullo trova riscontro nell'aspirazione di Dubois a vedere accresciuto il dominio della Francia fino al suo predominio in Europa.

Entrambi hanno inoltre avuto a cuore la diffusione del cristianesimo, cosi come entrambi sottolineano l'importanza dello studio delle lingue per ridisegnare l'idea di crociata, anche se in Lullo, che pubblicò il suo Liber de acquisitione Terre Sancte nel 1309, non viene esplicitata la contigua motivazione politica tanto importante in Dubois. 